\title{
Force Sensitive Resistance Based Heart Beat Monitoring For Health Care System
}

\author{
E.MuthuSundhara Pandiyan, M.Thamarai Selvan, M.Sadham Hussian and \\ Dr.G.Velmathi
}

Department of Electronics and Communication, Velammal College of Engineering and Technology, Viraganoor, Madurai-625009

\begin{abstract}
Heart related problems are the major health issues for the human. Almost 1 in every 4 death is mainly due to the heart problems. Most of the people death due to heart problems is due to poor monitoring.To address this issue this project has been designed. The main aim of this project is to measure the heart rate of an elderly people and to alert the care taker when the heart rate is abnormal. The innovation done in this project is measuring the force of the heartbeat at the wrist and thereby measuring the heart rate. The component used to measure this heart rate is FSR(Force Sensitive Resistor).This device can be implemented by means of wrist watch or wrist band. It also monitors the heart rate of the people for 24 hours.
\end{abstract}

\section{INTRODUCTION}

In recent scenario the continuous monitoring of patient becomes very essential as problem can arise at any point of time. The device should also be adoptable and compactable for the patients. Towards this aim a new device which continuously monitors the heartbeat of the patient and reports to the monitoring unit have been designed as a wearable device by which the patient feel better to wear. The monitoring unit monitors the heartbeat and if it crosses the threshold level it creates an alarm to alert the care takerthat particular patient's heart rate is abnormal. The monitoring unit will have a digital display to show the heart rate of the patient.

\section{HEART RATE FOR DIFFERENT AGE GROUP}

\begin{tabular}{|l|l|}
\hline Age & $\begin{array}{l}\text { Resting Heart } \\
\text { rate(beats/minute) }\end{array}$ \\
\hline 0-1 month & $70-190$ \\
\hline 1-11 months & $80-160$ \\
\hline 1-2 years & $80-130$ \\
\hline 3-4 years & $80-120$ \\
\hline 5-6 years & $75-115$ \\
\hline 7-9 years & $70-110$ \\
\hline Above 10 years & $60-100$ \\
\hline $\begin{array}{l}\text { Adults and Well } \\
\text { trained athletes }\end{array}$ & $40-60$ \\
\hline
\end{tabular}

Table 1: Heart rate for different age group 
International Journal of Information Sciences and Techniques (IJIST) Vol.4, No.3, May 2014

\section{EXISTING HEARTBEAT MEASURING TECHNIQUES}

\section{Infrared Sensor Technique:}

In this technique the heart rate of the patient is measured by means of Infrared diode and a photo diode as shown in figure1.

The major disadvantage of this technique is that it is temperature sensitive. There will be a change in Heart rate because of temperature. This technique works well only under certain temperature.

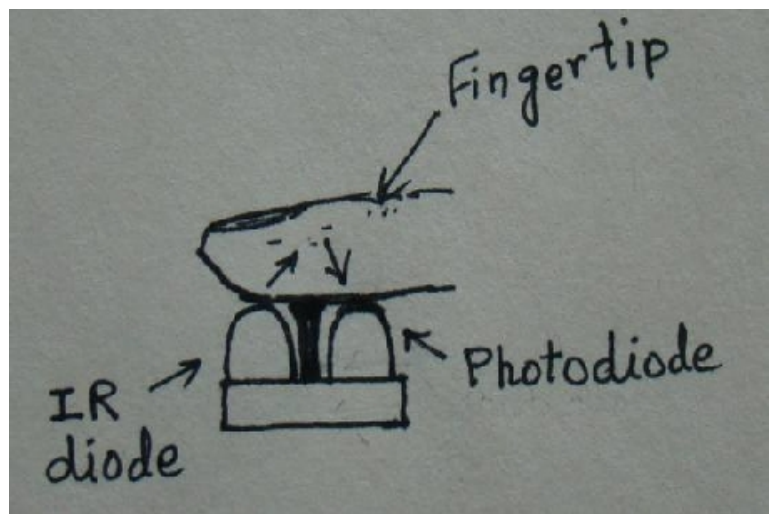

Figure 1: Infrared sensing technique

\section{Stethoscope method:}

The stethoscope is an acoustic medical device for auscultation, or listening to the internal sounds of an animal or human body. It is often used to listen to lung and heart sounds. This could not be used for monitoring the heart beat throughout the day.

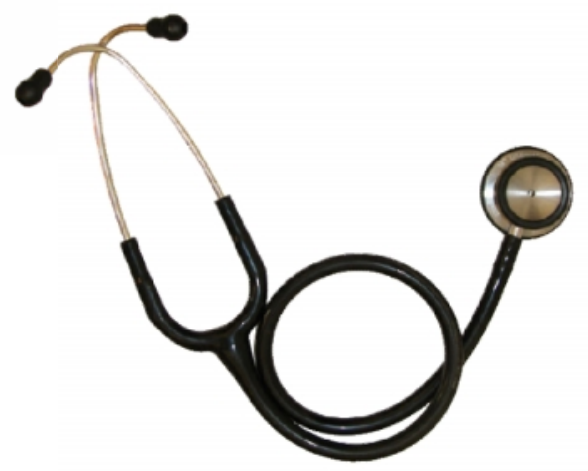

Figure 2: modern stethoscope

\section{PROPOSED METHOD}

Our proposed method is to monitor the heartbeat of the patient with the help of Force Sensitive Resistor. This system is used to monitor the patient continuously. In this system FSR detects the force of the heartbeat and the signal is given to the Microcontroller and the microcontroller counts the heartbeat. Then the Heartbeat of the patient is given to the Encoder (HT12E) and then is transmitted by wireless communication. The transmitted signal is received by the Decoder 
International Journal of Information Sciences and Techniques (IJIST) Vol.4, No.3, May 2014

(HT12D). The decoded signal is given to the microcontroller. This microcontroller sends the signal to the Display unit which displays the heart rate of the patient and also displays whether the pulse is normal or abnormal. It also makes the alarm circuit to give the alarm to alert the caretaker when the heartbeat is abnormal.

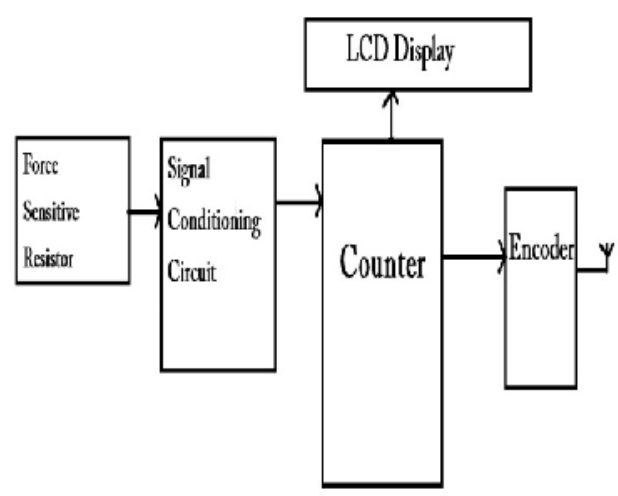

Figure 3: block diagram of transmitter

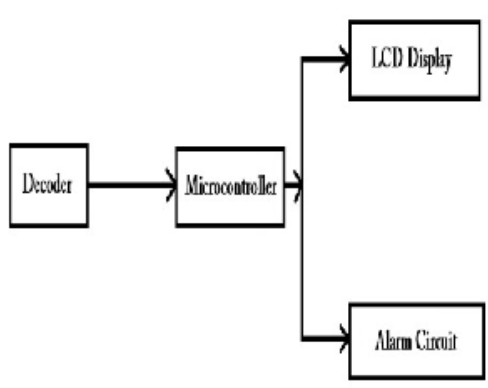

Figure 4: Block Diagram of Receiver

\section{SIMULATION}

Before implementing the actual system it's always beneficial to model and simulate the same for better performance and to save time in fabrication. The main aim of simulation in this workis to optimize the design of the heart pulse sensor.

It is planned to use $\mathrm{COMSOL}^{\mathrm{TM}}$ software for this simulation. COMSOL is the most convenient MEMS design tool used in the industry. It is an efficient design technique that reduces design risk, high speed and lower the development cost.

This simulation Tool has the following elements:

$>$ Layout editor: To draw the 2D, 3D structures.

$>$ Physical boundary defining aspect.

$>$ Material Database for mostly used MEMS material properties. 
$>$ Pre-processor: Creates 3D meshed layers based on solid model.

$>$ Post Process Analysis: Cross section plot parameters.

$>$ Complete report generating facility.

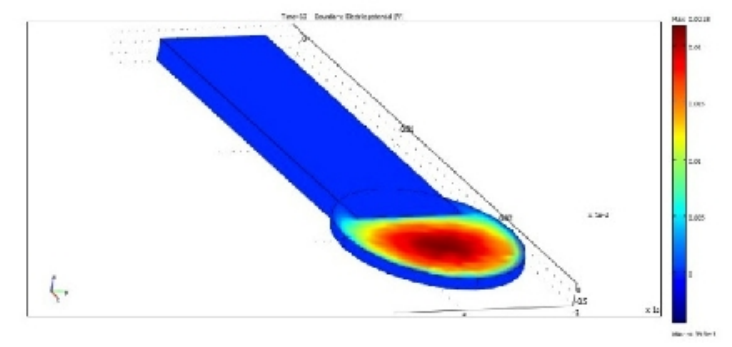

Figure 5: Simulation output

\section{Force Sensitive Resistor (FSR)}

The main component in this work is FSR. Force sensitive resistor is basically a resistor that will change its resistive value depending on the force of the heartbeat. This sensor is of low cost and easy to use.

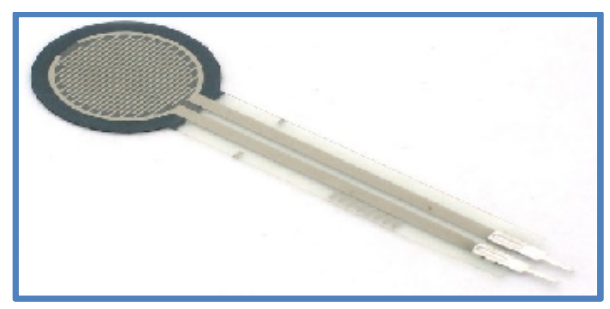

Figure 6: Force sensitive resistor

There will be a change in resistance with respect to the force of the heartbeat. When heartbeat occurs there will be increase in the force. This increase in force will reduce the resistance in FSR and this reduction in resistance will allow conductance and this conduction is counted as one heartbeat. This is the process involved in measuring the heart rate.

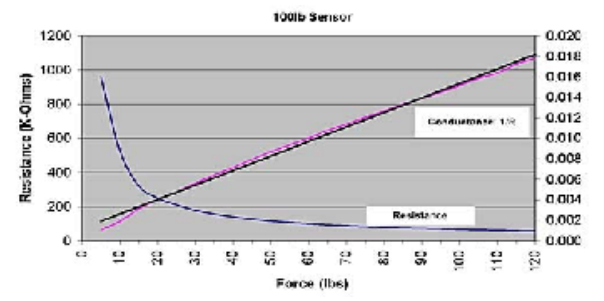

Figure 7: Force vs Resistance\& Conductance Characteristics

\section{Signal Conditioning}

Signal conditioning means improving an analog signal in such a way that it meets the required threshold values to be used in the next stage for the further processing The process involved in Signal Conditioning are

$>$ Amplification

$>$ Filtration 
$>$ Isolation

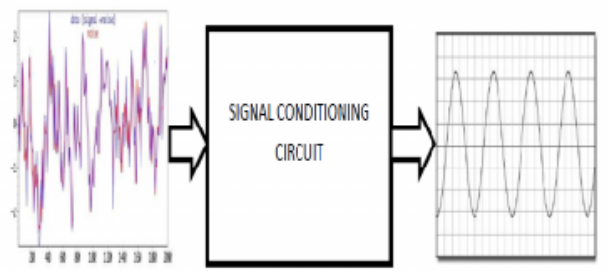

Figure 8: Block diagram for conditioning circuit

The bio signals have very low amplitude and noise in the envelope of the signal. Hence it needs to be processed before using it for further process. In conditioning first would be amplification of those signals with amplifiers such using op-amps or using BJTs. The unwanted signal frequencies could be removed using filters. Filtration is done so that band of frequencies with noise could be removed.

\section{Wireless Module}

The Technologies used for wireless module are

$$
\begin{aligned}
& >\text { Bluetooth } \\
& >\text { Wi-Fi } \\
& >\text { Wi-Max } \\
& >\text { RF module }
\end{aligned}
$$

\section{RF MODULE}

In this project we are going to use RF module for wireless transmission. Here weare using HT12E as a Transmitter module and HT12D is as a Receiver module.HT12E transmitter module uses $2^{12}$ series of encoders. These encoders begin a 4-word transmission cycle upon the receipt of transmission enable. This cycle will repeat itself as long as transmission enable is held low. Once the transmission enable returns high the encoder output completes its final cycle and stops.

HT12D receiver module uses $2^{12}$ series of decoders which provides various combinations of addresses and data pins in different packages so as to pair with the $2^{12}$ series of decoders. The decoders receive data that are transmitted by an encoder and interpret the first $\mathrm{N}$ bits of code period as address and the last $12-\mathrm{N}$ bits as data, where $\mathrm{N}$ is the address code number. A signal on the DIN pin activates the oscillator which in turn decodes the incoming address and data. The decoders will then check the received address three times continuously. If the received address codes all match the contents of decoders local address, the $12 \mathrm{~N}$-bit of data are decoded to activate the output pins and the VT pin is set high to indicate a valid transmission. This will last unless the address code is incorrect or no signal is received.

\section{CONCLUSION}

In this work, we have introduced concept of monitoring the heartbeat of the subjects remotely based on force sensitive resistor. This method is not sensitive towards temperature. By this method we can implement a system in which we could monitor a large number of subjects by a single care taker. We also use it as self-monitoring device. The objective of this project was to build a low power, low cost, reliable, non-intrusive and non-invasive monitoring system that 
International Journal of Information Sciences and Techniques (IJIST) Vol.4, No.3, May 2014

would accurately measure the vital signs. A reliable and continuous vital sign monitoring system targeted towards older individuals. It is also easy to use and provides accurate measurements.

\section{ACKNOWLEDGEMENT}

The authors thank Department of Science and Technology, Instrument Development Program (DST-IDP) for the financial support provided to this work.

\section{REFERENCES}

1. JunhoParl, InjeUniv, Gimhae, Jongman Cho, Junghyeon Choi, Taewoo Nam. A zigbee network-based multi-channel heart rate monitoring system for exercising rehabilitation patients. TENCON 20072007 IEEE Region 10 Conference

2. Engin, M. ; ElektrikveElektron. MuhendisligiBolumu, Ege Univ., Izmir ; Engin, E.Z. ; Bildik, S. ; Karipcin, T. Portable Heart Rate Monitoring System. Biomedical Engineering Meeting, 2009. BIYOMUT 2009. 14th National.

3. Dhvani Parekh.Designing Heart Rate, Blood Pressure and Body Temperature Sensors for Mobile OnCall System..EE 4BI6 Electrical Engineering Biomedical Capstones.Paper 39.

4. Yaofeng Wen ; Rong Yang ; Yuquan Chen.Heart rate monitoring in dynamic movements from a wearable system.Medical Devices and Biosensors, 2008.ISSS-MDBS 2008. 5th International Summer School and Symposium

5. Kioumars, A.H,Liqiong Tang Wireless network for health monitoring: heart rate and temperature sensor. SensiTechnology (ICST), 2011 Fifth International Conference.

6. Sornanathan, L, Khalil, I. Fitness monitoring system based on heart rate and $\mathrm{SpO} 2$ level. Information Technology and Applications in Biomedicine (ITAB), 2010 10th IEEE International Conference

\section{Authors}

E.Muthu Sundhara Pandiyan , Born in Theni, India on $14^{\text {th }}$ of October 1992. The author is pursuing Bachelor degree in Velammal College of Engineering and Technology, Madurai, Tamil Nadu, India. His research interests are sensor networks and VLSI design.

M.Thamarai Selvan, Born in Madurai, India on $3^{\text {rd }}$ of December 1991. The author is pursuing Bachelor degree in Velammal College of Engineering and Technology, Madurai, Tamil Nadu, India. His research interests are sensor networks and VLSI design.

M.Sadham Hussian, Born in Madurai, India on $6^{\text {th }}$ of February 1992. The author is pursuing Bachelor degree in Velammal College of Engineering and Technology, Madurai Tamil Nadu ,India. His research interests are Sensor networks and VLSI Design.

Dr.G.Velmathi is currently working as a professor in Electronics and Communication VCET and has teaching experience of 19 years. She has completed her B.E in Thiagarajar college of engineering and M.E in Madras Institute of Science and Technology. She Received her Ph.D from Indian Institute of Science as a full time research scholar and her are of interest is mainly RFMEMS, VLSI design and Biomedical instrumentation. She is the principal investigator of two ongoing DST

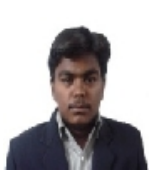
projects worth 78Lakhs. She authored many paper works in the field of MEMS and VLSI
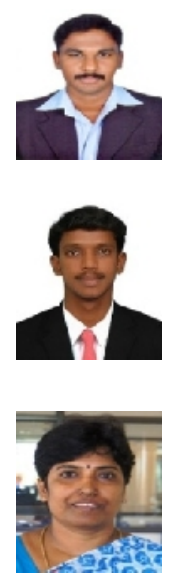